\title{
Un Nouveau Signalement de Plante du LIBAN
}

\author{
Georges Tohmé et Henriette Tohmé \\ CNRS BP 118281 Beyrouth, Liban \\ gtohme@ cnrs.edu.lb
}

(Received 4 October 2016 - Accepted 15 December 2016)

RÉSUMÉ

Tohmé, G. et Tohmé, H. 2016. Un nouveau signalement de plante du Liban. Lebanese Science Journal, 17(2): 240-241.

Dans cette note, les auteurs décrivent une Iris du Liban, signalée auparavant par Mouterde de Syrie. Ils l'avaient appelé Iris persica tauri, en 2014.

Mots clés: espèce nouvelle, flore sauvage, habitat.

\section{ABSTRACT}

Tohmé, G. and Tohmé, H. 2016. A new plant species in Lebanon. Lebanese Science Journal, 17(2): 240-241.

In this note, the authors give a description of an Iris from Lebanon cited previously from Syria by Mouterde. They have named it, in 2014, I. persica tauri.

Keywords: new specie, wild flora, habitat.

\section{INTRODUCTION}

Une thèse de doctorat sur la caractérisation génétique du genre Iris évoluant dans la Méditerranée orientale (Abdel Samad 2016), montre que l'iris de Qaa, signalée dans Illustrated Flora of Lebanon 2014, sous le nom de Iris persica var tauri (Siehe) Bornm. Est génétiquement différente de l'Iris persica. Les auteurs s'étaient basés sur la description de Thiebault (1953). Dans la présente note, elle correspondrait plutôt à Iris postii (Mouterde, 1966) qui provient du désert Syrien.

\section{LA NOUVELLE ESPECE DE QAA}

\section{Iris postii Mouterde (IRIDACEAE)}

L'iris de Qaa ressemble à l'Iris postii du désert syrien décrite par Mouterde en 1966. D'après Al-Eissawi (1998) elle se trouve aussi dans les terrains désertiques de Mafraq en Jordanie. Collenette (1999), la signale de l'Arabie Saoudite près de la frontière jordanienne sur

http://dx.doi.org/10.22453/LSJ-017.2.240241

National Council for Scientific Research - Lebanon 2016@

1sj.cnrs.edu.lb/vol-17-no-2-2016/ 
du sable couvrant l'argile. La description morphologique de ces trois auteurs est identique à l'iris de Qaa. Une étude génétique comparée des différentes I. postii, devrait être réalisée. Cette plante ressemble à Iris persica L. par: a/ son bulbe écailleux et ses racines charnues persistantes; $\mathrm{b}$ / par des tépales internes très petits et externes larges au milieu et rétrécis vers l'extrémité; $\mathrm{c} /$ par des feuilles arquées et étroites à marge blanche.

Les deux espèces différent chez I. postii par le bulbe plus ové et à spathe basale allongée, par la tige plus longue $(>20 \mathrm{~cm})$ et par le périanthe atteignant $5 \mathrm{~cm}$, d'un bleu violet intense et dont l'intensité baisse avec la croissance (Figure 1).

Les auteurs ont observé cette plante pour la première fois le 31 décembre 2010. Elle était au début de sa floraison, puis le 2 février 2011. La région de Qaa, au nord de la Beqaa est réputée pour son climat sub-désertique, proche de celui du désert syrien. Le terrain qui accueille cet iris était sec depuis plus de 2 mois. L'accès à ce terrain n'était pas aisé et généralement le taux de la pluviométrie annuelle ne dépasse pas $250 \mathrm{~mm}$.

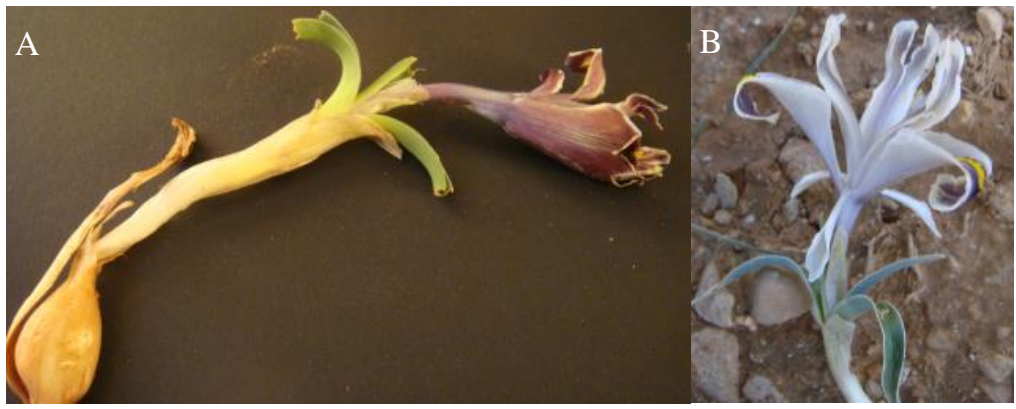

Figure 1. A) Iris postii Mt bulbe ovoide et spathe allongée B) Iris postii de Qaa

\section{CONCLUSION}

En conclusion, cette note fait suite à celle des auteurs en 2015, où il est dit que de nouvelles plantes sauvages continuent à être signalées au Liban pendant que d'autres s'éteignent suite au développement de l'agriculture en montagne et à l'intérieur du pays et de l'urbanisation de la région côtière.

\section{RÉFÉRENCES}

Abdel Samad, N. 2016. Caractérisation génétique du genre Iris évoluant dans la Méditerranée orientale. Thèse de doctorat, université Paris Sacley-France et université Saint Joseph de Beyrouth-Liban.

Al-Eissawi, D. 1998. Wild flowers of Jordan and neighboring countries. Amman: J. P. F.

Collenette, Sh. 1999. Wild flowers Saudi Arabia. NCWCD, Kingdom of Saudi Arabia.

Mouterde, P. 1966. Nouvelle Flore du Liban et de la Syrie. 1er tome, Dar-el-Machreq/Publisher, Beyrouth.

Thiebaut, J. 1953. Flore libano-syrienne (3e partie). Editions du CNRS-Paris.

Tohmé, G. et Tohmé, H. 2014. Illustrated Flora of Lebanon. CNRS, Liban.

Tohmé, G. et Tohmé, H. 2015. Nouveaux signalements de plantes du Liban. Lebanese Science Journal, 16 (2): 139-141. 